\title{
Targeting Angiogenesis in Advanced Non-Small Cell Lung Cancer
}

\author{
Philip E. Lammers, MD, MSCI and Leora Horn, MD, MSc \\ Vanderbilt Ingram Cancer Center, Vanderbilt University, Nashville, TN
}

\begin{abstract}
Lung cancer is the leading cause of cancer-related mortality in the United States. Over the past 40 years, treatments with standard chemotherapy agents have not resulted in substantial improvements in long-term survival for patients with advanced lung cancer. Therefore, new targets have been sought, and angiogenesis is a promising target for non-small cell lung cancer (NSCLC). Bevacizumab, a monoclonal antibody targeted against the vascular endothelial growth factor, is the only anti-angiogenic agent that is currently recommended by the National Comprehensive Cancer Network (NCCN) for the treatment of advanced NSCLC. However, a number of antibody-based therapies and multi-targeted tyrosine kinase inhibitors are currently under investigation for the treatment of patients with NSCLC. This review summarizes the available clinical trial data on the efficacy and safety of these agents in advanced lung cancer patients.
\end{abstract}

\section{Keywords}

angiogenesis; non-small cell lung cancer; bevacizumab; adverse events

\section{Introduction}

Lung cancer is the leading cause of cancer-related mortality in the United States with an estimated 228,190 new cases and an estimated 159,480 deaths in 2013. ${ }^{1}$ Over two-thirds of patients with lung cancer will present with advanced disease. ${ }^{2}$ The 5 -year survival rate is approximately $15 \%$. Standard platinum-based chemotherapy regimens are associated with survival of approximately 1 year in patients with advanced lung cancer. ${ }^{3}$ Approximately $60 \%$ of patients with stage IIIB/IV adenocarcinoma have a molecular mutation thought to drive tumor growth. ${ }^{4}$ However, only those patients with epidermal growth factor receptor (EGFR) mutations (approximately 10-15\%) or anaplastic lymphoma kinase (ALK) rearrangements (approximately 5\%) have a Food and Drug Administration (FDA)-approved therapy available. Other potential targets such as c-ros oncogene 1 (ROS1) gene fusions and

Please address all correspondence and reprint requests to: Leora Horn, MD, MSc, Assistant Professor, Vanderbilt Ingram Cancer Center, Vanderbilt University, 2220 Pierce Avenue, 777 Preston Research Building, Nashville, TN 37232, Phone: 615-936-3524, Fax: 615-343-7602, leora.horn@vanderbilt.edu.

Conflicts of Interest

The authors have no potential conflicts of interest to disclose.

Note: For consistency, efficacy data have been converted to months using the following: 1 month $=4.2$ weeks for data reported in weeks; 12 months $=365$ days for data reported in days. For consistency, where necessary, PFS and OS values have been rounded to the nearest tenth, and HR, CI, and $P$ values have been rounded to 2 decimal places. 
BRAF mutations have been identified and clinical trials using targeted agents are ongoing. Alternative targets continue to be investigated, one of which is angiogenesis, a necessary step in the growth and metastasis of solid tumors. ${ }^{5}$ Bevacizumab is the only anti-angiogenic therapy FDA-approved for NSCLC, ${ }^{6}$ but other agents have been tested in advanced NSCLC. At the present time, the National Comprehensive Cancer Network (NCCN) recommends consideration of the use of bevacizumab in the first-line treatment setting for stage IV disease in combination with a platinum doublet with continuation of bevacizumab until progression. The use of anti-angiogenic agents is a rational approach to treat lung cancer but needs to be balanced against the potential risks involved, which can be life-threatening. Researchers have been searching for potential biomarkers to identify patients for whom therapy with anti-angiogenic inhibitors may be most beneficial. The current data for antiangiogenic agents varies widely among the studied drugs, and safety data are especially limited for many of the agents studied. This review covers efficacy and safety/tolerability data from clinical trials of anti-angiogenic agents in advanced NSCLC.

\section{Efficacy and Safety of Anti-angiogenic Agents for NSCLC}

\section{Antibody-Based Therapeutics}

Bevacizumab-Bevacizumab is a humanized monoclonal antibody with a high affinity for vascular endothelial growth factor (VEGF). ${ }^{7}$ Bevacizumab binds to circulating VEGF, preventing it from binding to the VEGF receptor (VEGFR) and thereby inhibiting downstream signaling. The sites of action of bevacizumab and other anti-angiogenic agents described in the text are depicted in Figure 1. Bevacizumab has been studied extensively in various malignancies and certain adverse events (AEs), such as bleeding and thrombosis, are known to be associated with its use. In addition, hypertension and proteinuria are common throughout treatment, although these are generally manageable with anti-hypertensive therapies.

Bevacizumab is the most studied anti-angiogenic agent in advanced NSCLC (Table 1 and 2). Following promising results from a phase II study, ${ }^{8}$ ECOG 4599 was conducted as a randomized phase III trial that compared carboplatin/paclitaxel with or without bevacizumab in 878 patients with recurrent or advanced nonsquamous NSCLC. ${ }^{9}$ Improvements in median overall survival (OS), median progression-free survival (PFS), and response rate (RR) occurred in bevacizumab-treated patients compared with the chemotherapy arm: 12.3 versus 10.3 months, 6.2 versus 4.5 months, and 35\% versus $15 \%$, respectively. In an unplanned subset analysis, median PFS and RR were significantly improved with bevacizumab versus chemotherapy for both sexes; however, median OS was not improved in the female cohort but was improved among men (11.7 vs 8.7 months with chemotherapy). ${ }^{10}$ In another unplanned subset analysis, elderly (age $\geq 70$ years) patients had improved median PFS and RR, but no improvement in median OS. ${ }^{11}$ The most common grade $\geq 3$ AEs in the bevacizumab arm were neutropenia (26\%), hypertension (7\%), febrile neutropenia (5\%), and bleeding events (4\%). ${ }^{9}$ Compared with chemotherapy alone, bevacizumab plus chemotherapy was associated with higher rates of grade 4 neutropenia ( $26 \%$ vs $17 \%$ ), grade 4 thrombocytopenia ( $1.6 \%$ vs $0.2 \%$ ), and grade $3 / 4$ febrile neutropenia ( $4.0 \%$ vs $1.8 \%$ ), 
hyponatremia (3.5\% vs $1.1 \%)$, hypertension $(7.0 \%$ vs $0.7 \%)$, headache $(3.0 \%$ vs $0.5 \%)$, rash or desquamation $(2.3 \%$ vs $0.5 \%)$, and bleeding events $(4.4 \%$ vs $0.7 \%)$.

The AVAIL trial was a similarly designed phase III trial conducted in Europe and Canada to evaluate the efficacy of cisplatin and gemcitabine with or without bevacizumab (7.5 or 15 $\mathrm{mg} / \mathrm{kg}$ ) in 1,043 patients with advanced or recurrent nonsquamous NSCLC. ${ }^{12}$ Median PFS (6.7 months in bevacizumab $7.5 \mathrm{mg} / \mathrm{kg}$ arm and 6.5 months in bevacizumab $15 \mathrm{mg} / \mathrm{kg}$ arm vs 6.1 months in placebo arm) and RR (38\% in bevacizumab $7.5 \mathrm{mg} / \mathrm{kg}$ arm and $35 \%$ in bevacizumab $15 \mathrm{mg} / \mathrm{kg}$ arm vs $22 \%$ in placebo arm) were significantly improved in both bevacizumab-containing arms, but OS was not improved in either bevacizumab-containing arm compared with placebo (13.6 months, 13.4 months, and 13.1 months, respectively). ${ }^{13}$ Grade 3/4 AEs in the bevacizumab $15-\mathrm{mg} / \mathrm{kg}$ arm included hypertension (9\%), vomiting (9\%), neutropenia (36\%), bleeding (4\%), and proteinuria (1\%). Grade 3/4 AEs in the bevacizumab $7.5-\mathrm{mg} / \mathrm{kg}$ arm included hypertension $(6 \%)$, vomiting (7\%), neutropenia $(40 \%)$, bleeding $(4 \%)$, and proteinuria $(<1 \%)$. Pulmonary hemorrhage was observed in $1.5 \%$ of patients in the bevacizumab $7.5-\mathrm{mg} / \mathrm{kg}$ arm, $0.9 \%$ in the bevacizumab $15-\mathrm{mg} / \mathrm{kg}$ arm, and $0.6 \%$ in the placebo arm.

A phase II study of pemetrexed/carboplatin/bevacizumab followed by maintenance pemetrexed and bevacizumab showed impressive results ${ }^{14}$ and led investigators to conduct a large randomized phase III trial of 939 patients to evaluate for superiority of pemetrexed/ carboplatin/bevacizumab followed by pemetrexed/bevacizumab maintenance compared with paclitaxel/carboplatin/bevacizumab followed by single-agent bevacizumab maintenance. ${ }^{15}$ Only PFS was statistically superior in the pemetrexed/carboplatin/bevacizumab arm (6.0 vs 5.6 months for paclitaxel/carboplatin/bevacizumab), but RR (34.1\% vs 33.0\%) and OS (12.6 vs 13.4 months) did not show superiority. The toxicities differed between arms; there was more grade $3 / 4$ thrombocytopenia $(23.3 \%$ vs $5.6 \%$ ), anemia ( $14.5 \%$ vs $2.7 \%$ ), and fatigue $(10.9 \%$ vs $5.0 \%)$ in the pemetrexed group, whereas there was more grade $3 / 4$ neutropenia $(40.6 \%$ vs $25.8 \%)$, febrile neutropenia ( $4.1 \%$ vs $1.4 \%)$, and sensory neuropathy (4.1\% vs $0 \%)$ in the paclitaxel group.

Several studies have evaluated bevacizumab in patients who have historically been excluded from other trials. The phase II BRIDGE trial studied carboplatin/paclitaxel and delayed bevacizumab in 31 previously untreated patients with advanced squamous NSCLC. ${ }^{16}$ Efficacy results have not been published, but the 4 most common grade 3/4 AEs were hypertension (16\%), dyspnea (10\%), deep vein thrombosis (7\%), and arthralgia (7\%). One patient had grade $\geq 3$ pulmonary hemorrhage and another had grade 1 pulmonary hemorrhage.

The phase II BRAIN trial evaluated the safety of bevacizumab given in the first-line setting with carboplatin/paclitaxel or in the second-line setting in combination with erlotinib in patients with nonsquamous NSCLC and asymptomatic, untreated brain metastases. Grade 1 intracranial hemorrhage occurred in 1 of 67 patients in the first-line setting and 0 of 24 patients in the second-line setting, and the RR for intracranial metastases was $61 \%$ in firstline therapy and $21 \%$ in second-line therapy. ${ }^{17}$ 
Ramucirumab-Ramucirumab (IM-1121B), a human monoclonal anti-VEGFR-2 antibody, ${ }^{18}$ is currently being evaluated in patients with diverse histological subtypes of NSCLC, including those with squamous cell histology and/or treated brain metastases. Results from a single-arm phase II trial of 40 patients treated with carboplatin, paclitaxel, and ramucirumab reported a RR of 55\% and median PFS of 7.9 months. ${ }^{19}$ Grade 3/4 AEs included thrombocytopenia (10\%), febrile neutropenia (7.5\%), peripheral neuropathy and pulmonary embolism (5\% each).

In a separate phase II randomized study in patients with nonsquamous NSCLC, ramucirumab plus pemetrexed was given in combination with either carboplatin or cisplatin versus single-agent pemetrexed in combination with carboplatin or cisplatin. ${ }^{20} \mathrm{An}$ interim analysis showed a RR of $44 \%$ and PFS of 6.3 months in the ramucirumab arm versus a RR of $37 \%$ and PFS of 4.3 months in the chemotherapy alone arm. Grade 3 AEs in the ramucirumab arm included thrombocytopenia (15\%), neutropenia (13\%), fatigue (12\%), and nausea $(10 \%)$.

Bavituximab-Bavituximab is a monoclonal antibody against phosphatidylserine that causes selective shutdown of existing tumor blood vessels. ${ }^{21}$ A randomized phase II study of 86 patients with nonsquamous histology compared carboplatin/paclitaxel with or without bavituximab. $^{22}$ In the bavituximab group, the RR was $32 \%$ and PFS was 5.8 months, and in the chemotherapy alone group, the RR was $31 \%$ and PFS was 4.6 months. OS was not yet reached at the time of reporting. The most common grade $3 / 4$ AEs were anemia (6.8\% with bavituximab vs $7.1 \%$ with chemotherapy alone), neutropenia ( $6.8 \%$ vs $9.5 \%$ ), and thrombocytopenia ( $6.8 \%$ vs $2.4 \%)$.

Aflibercept-Aflibercept (AV0005), an angiogenesis inhibitor composed of portions of the extracellular domains of human VEGFR-1 and VEGFR-2 fused to the Fc portion of human immunoglobulin G, is currently being evaluated in NSCLC. ${ }^{23}$ In a single-arm, phase II trial, aflibercept was administered to 98 patients with platinum- and erlotinib-resistant lung adenocarcinoma, and results showed a RR of $2 \%$, median PFS of 2.7 months, and median OS of 6.2 months. ${ }^{24}$ Most common grade 3/4 AEs were hypertension (23\%), dyspnea $(21 \%)$, proteinuria (10\%), and fatigue (7\%). A phase III trial (VITAL) of docetaxel plus aflibercept vs docetaxel alone as second-line therapy in advanced NSCLC showed an improvement in RR (23\% vs 9\%), median PFS (5.2 months vs 4.1 months), but OS was not improved (10.1 months vs 10.4 months). ${ }^{25}$ The most common grade $3 / 4$ AEs were neutropenia ( $28 \%$ in aflibercept arm vs $21 \%$ in chemotherapy alone arm), fatigue ( $11 \%$ vs $4 \%)$, and stomatitis ( $9 \%$ vs $1 \%$ )

\section{Tyrosine Kinase Inhibitors}

Resistance to VEGF inhibition has been shown to be multi-factorial. ${ }^{26}$ Receptor tyrosine kinase inhibitors (TKIs), many of which target several angiogenesis pathways, are a class of agents in clinical development for various malignancies. Many of the multi-targeted agents will theoretically inhibit several angiogenesis pathways and may specifically overcome resistance to VEGF inhibition. Several of these multi-targeted TKIs have been investigated for use in the treatment of NSCLC in clinical trials. 


\section{Sorafenib}

Sorafenib is a multi-targeted TKI that inhibits VEGFR-2, VEGFR-3, platelet-derived growth factor (PDGF) receptor- $\beta$ (PDGFR- $\beta$ ), v-raf1 murine leukemia viral oncogene homolog 1 (Raf), fms-like tyrosine kinase 3 (FLT-3), and stem cell factor receptor (c-KIT) ${ }^{27}$ Sorafenib showed single-agent activity in several phase II trials in patients with previously treated advanced nonsquamous NSCLC, ${ }^{28,29}$ but large randomized phase III trials have been disappointing. ${ }^{30,31}$ A phase III trial (ESCAPE) of 926 patients with advanced nonsquamous and squamous cell NSCLC was halted due to lack of efficacy on interim analysis. ${ }^{30}$ Patients with squamous histology receiving sorafenib had a shorter median OS (8.9 vs 13.7 months) compared with patients receiving chemotherapy alone. The 4 most common grade 3/4 AEs in the sorafenib arm were neutropenia (9\%), rash/desquamation (8\%), hand-foot skin reaction $(8 \%)$, and fatigue $(5 \%)$, while in the chemotherapy arm, these were neutropenia $(6 \%)$, fatigue (3\%), and diarrhea, sensory neuropathy, vomiting, and nausea ( $2 \%$ each). Four of the 6 fatal hemorrhagic/bleeding events observed in the study occurred in patients with squamous histology ( 2 in each arm).

A second phase III trial (NEXUS) excluded patients with squamous cell histology, subsequent to a protocol amendment. ${ }^{31}$ This trial combined cisplatin/gemcitabine with or without sorafenib in 904 patients with advanced NSCLC, showing no difference in median OS with sorafenib versus placebo in nonsquamous disease (12.4 vs 12.5 months) but a statistically significant increase in median PFS (6.0 vs 5.5 months). Reported grade $\geq 3$ AEs attributable to sorafenib included thrombocytopenia (10\%), hand-foot skin reaction (9\%), fatigue $(7 \%)$, and rash (6\%).

\section{Sunitinib}

Sunitinib is a multi-targeted TKI that inhibits VEGFR-2, PDGFR- $\beta$, rearranged during transfection (RET), c-KIT, and FLT- $3 .^{32}$ Sunitinib has shown single-agent activity in phase II trials in previously treated NSCLC patients. ${ }^{33,34}$ A phase III trial of sunitinib plus erlotinib versus erlotinib alone as second- or third-line therapy in 960 patients (90\% with unknown EGFR mutational status) showed no significant difference between groups in the primary endpoint of OS (9.0 vs 8.5 months). ${ }^{35}$ The most common grade $3 / 4$ toxicities with sunitinib plus erlotinib were rash/dermatitis (17\%), diarrhea (16\%), and hypophosphatemia $(13 \%)$, all higher than with erlotinib alone (10\%, 3\%, and $4 \%$, respectively).

\section{Nintedanib}

Nintedanib (BIBF 1120) is a multi-targeted TKI that targets VEGFR-1, VEGFR-2, VEGFR-3, PDGFR- $\alpha$, PDGFR- $\beta$, fibroblast growth factor (FGF) receptor (FGFR)-1, FGFR-2, and FGFR-3; in addition, nintedanib has activity against FLT-3 and the v-src sarcoma viral oncogene homolog (src) family. ${ }^{36}$ A phase II study of nintedanib dosed either at $250 \mathrm{mg}$ twice daily or $150 \mathrm{mg}$ twice daily in 73 patients with relapsed NSCLC showed mild activity. ${ }^{37}$ A phase III study (LUME-Lung 1) in 1314 patients with advanced or metastatic squamous and non-squamous NSCLC that had progressed on first-line chemotherapy randomized patients to nintedanib or placebo in combination with docetaxel. 38 There was an improvement in median PFS (3.4 months vs 2.7 months), but not in median OS (10.1 months vs 9.1 months) in the nintedanib/docetaxel arm vs docetaxel/placebo arm. 
Grade 3/4 AEs were similar in each arm, the most common included elevated ALT (8\% vs $1 \%$ ), and diarrhea (7\% vs 3\%). A separate phase III study (LUME-Lung 2) in patients with advanced or metastatic non-squamous NSCLC that had progressed on first-line chemotherapy comparing nintedanib or placebo in combination with pemetrexed was stopped early because of a signal for futility on an interim analysis. ${ }^{39}$ The analysis of 713 enrolled patients (initially planned to enroll 1300 patients) showed an increase in median PFS (4.4 months vs 3.6 months) but no difference in RR (9\% vs 9\%) or median OS (HR 1.03). Reported grade $3 / 4$ AEs included elevated ALT ( $23 \%$ vs $7 \%$ ), elevated AST ( $12 \%$ vs $2 \%)$, and diarrhea (3\% vs $1 \%)$.

\section{Cediranib}

Cediranib inhibits VEGFR-1, VEGFR-2, VEGFR-3, PDGFR- $\alpha / \beta$, FGFR-1, and c-KIT. ${ }^{40} \mathrm{~A}$ phase II/III trial comparing carboplatin/paclitaxel with or without cediranib $30 \mathrm{mg}$ in 296 patients with advanced NSCLC was halted early due to imbalances in the number of deaths observed in cediranib-treated patients. ${ }^{41}$ The RR was $38 \%$, median PFS was 5.6 months, and median OS was 10.5 months in the cediranib group compared with a RR of $16 \%$, median PFS of 5.0 months, and median OS of 10.1 months in the placebo group. The 4 most common grade $3 / 4 \mathrm{AEs}$ in the cediranib arm were neutropenia (49\%), fatigue (29\%), increased thyroid-stimulating hormone (TSH; 27\%), and hypertension (19\%). A phase II trial evaluating pemetrexed and cediranib in 2 cohorts of patients (a bevacizumab-naive group and a bevacizumab-pretreated group) has completed accrual of bevacizumab-naive patients. ${ }^{42}$ Preliminary results in the bevacizumab-naive group showed a RR of $29 \%$, median PFS of 5.6 months, and median OS of 11 months. The 4 most common grade 3/4 AEs in the bevacizumab-naive cohort were fatigue (22\%), neutropenia (14\%), diarrhea (14\%), and infection $(8 \%)$. Three treatment-related deaths have been reported.

\section{Motesanib}

Motesanib is a multi-targeted TKI that targets VEGFR-1, VEGFR-2, VEGFR-3, PDGFR- $\beta$, c-KIT, and RET. ${ }^{43}$ A phase II trial of motesanib in combination with doublet chemotherapy ${ }^{44}$ as well as a phase III trial of carboplatin and paclitaxel with or without motesanib (MONET1) were performed. The phase III study was initially suspended due to a higher incidence of hemoptysis and mortality in patients with squamous cell histology. The trial resumed in patients with only nonsquamous histology and did not show a statistically significant improvement in median OS. ${ }^{45}$ The RR was $40 \%$, median PFS was 5.6 months, and median OS was 13.0 months in the motesanib arm versus 26\%, 5.4 months, and 11.0 months, respectively, in the placebo arm. Grade $\geq 3$ AEs with motesanib included neutropenia ( $22 \%$ vs $15 \%$ with placebo), diarrhea (9\% vs $1 \%$ ), hypertension ( $7 \%$ vs $1 \%$ ), and cholecystitis ( $3 \%$ vs $0 \%$ ). The incidence of grade 5 AEs was $14 \%$ with motesanib versus $9 \%$ with placebo.

\section{Pazopanib}

Pazopanib is a multi-targeted TKI that inhibits VEGFR-1, VEGFR-2, VEGFR-3, PDGFR-a/ $\beta$, FGFR-1, FGFR-3, and c-KIT, ${ }^{46}$ and is currently being evaluated. In a phase II trial, 192 patients with advanced NSCLC who had failed 1 to 2 prior lines of therapy were randomized to pazopanib plus erlotinib versus placebo plus erlotinib. ${ }^{47}$ There was a statistically 
significant improvement in PFS in the combination arm (2.6 vs 1.8 months with erlotinib alone) but similar RR ( $6 \%$ vs $0 \%$ ) and OS (6.8 vs 6.7 months) in the two arms. Severe nonhematologic toxicities in the combination group were fatigue (20\%), diarrhea (19\%), and proteinuria $(5 \%)$.

\section{Axitinib}

Axitinib is a multi-targeted TKI that targets VEGFR-1, VEGFR-2, VEGFR-3, PDGFR- $\beta$, and c-KIT. ${ }^{48}$ A phase II study of axitinib in 32 patients with NSCLC showed a RR of $9 \%$, median PFS of 4.9 months, and median OS of 14.8 months. ${ }^{49}$ Grade 3 hypertension (9\%) and diarrhea and vomiting ( $3 \%$ each) were reported. In a randomized phase II study of 2 dosing schedules (continuous or intermittent) of axitinib with first-line pemetrexed/cisplatin in 170 patients with nonsquamous NSCLC, the axitinib arms were associated with higher RRs versus chemotherapy alone (45.5\% in the continuous arm and $39.7 \%$ in the intermittent arm vs $26.3 \%$ in the chemotherapy alone arm) but with no significant prolongation of PFS (8.0, 7.9, and 7.1 months, respectively) or OS (16.6, 14.7, and 15.9 months, respectively). ${ }^{50}$ The most common grade 3 AEs were hypertension (20\%), neutropenia (18\%), and nausea (16\%) with continuous axitinib and hypertension (17\%), fatigue (16\%), and anemia (14\%) with intermittent axitinib, with reports of grade 4 asthenia (1\%) and pulmonary embolism (1\%) with the latter schedule.

A phase II randomized study of axitinib or bevacizumab combined with paclitaxel/ carboplatin as first-line therapy for patients with nonsquamous NSCLC failed to show an improvement with axitinib compared with bevacizumab in RR ( $29 \%$ vs $43 \%$, respectively), PFS (5.7 vs 6.1 months), or OS (10.6 vs 13.3 months). The most common grade $3 / 4 \mathrm{AE}$ in both arms was neutropenia, and there was a higher rate of treatment discontinuation due to AEs with axitinib than with bevacizumab ( $41 \%$ vs $31 \%$, respectively). ${ }^{51}$

\section{Vandetanib}

Vandetanib is a TKI that inhibits VEGFR signaling, EGFR signaling to a lesser extent, and RET tyrosine kinases. ${ }^{52}$ Vandetanib is no longer in development for the treatment of NSCLC. Several phase III trials failed to show a significant improvement in OS among previously treated patients with advanced NSCLC when vandetanib was combined with chemotherapy (ZEAL, ZODIAC), ${ }^{53,54}$ given as a single-agent after failure of an EGFR TKI (ZEPHYR), ${ }^{55}$ or compared with erlotinib (ZEST). ${ }^{56}$

\section{Linifanib}

Linifanib (ABT-869) is a multi-targeted TKI that is being evaluated in NSCLC. It inhibits VEGFR-1, VEGFR-2, VEGFR-3, PDGFR- $\beta$, c-KIT, CSF-1R, and FLT-3. ${ }^{57}$ Results from a phase II trial in 139 patients evaluating 2 doses of linifanib in chemo-refractory NSCLC showed a RR of 5.0\%, median PFS of 3.6 months, and median OS of 9.0 months overall. ${ }^{58}$ The incidence of grade $3 / 4$ hypertension was $1.5 \%$ in the $0.1-\mathrm{mg} /$ day group and $24.3 \%$ in the $0.25-\mathrm{mg} /$ day group; no other grade $3 / 4$ AEs were observed in $>10 \%$ of patients overall. 


\section{Vascular Disrupting Agents}

\section{Ombrabulin}

Ombrabulin (AVE8062) is a vascular disrupting agent and analog of combretastatin A4 that damages tumor vasculature. ${ }^{59}$ The phase II DISRUPT trial randomized 176 patients with either squamous or nonsquamous histology to therapy with ombrabulin or placebo combined with a chemotherapy backbone of either cisplatin/docetaxel or carboplatin/paclitaxel for 6 cycles. ${ }^{60}$ The RR was $32 \%$ in the ombrabulin arm versus $31 \%$ in the placebo group, PFS was 5.7 versus 5.5 months, and OS was 11.0 months in each arm. The safety profile was reported to be similar with 57\% unspecified grade 3/4 AEs in the ombrabulin arm versus $52 \%$ in the placebo arm.

\section{Vadimezan}

Vadimezan (ASA404) is a vascular disrupting agent of the flavonoid class. ${ }^{61}$ After promising results in a phase II trial in untreated patients, ${ }^{62}$ it was tested in a phase III trial in advanced or metastatic NSCLC in combination with carboplatin/paclitaxel vs carboplatin/ paclitaxel alone. 1299 patients were enrolled and the trial was stopped early due to futility. There was no statistical difference in OS (13.4 months vs 12.7 months), PFS (5.5 months vs 5.5 months), or RR ( $25 \%$ vs $25 \%$ ) in the vadimezan arm vs chemotherapy alone. ${ }^{63}$

\section{Lack of Predictive Biomarkers for Anti-Angiogenic Therapy in Lung Cancer}

As summarized in this review, many anti-angiogenic agents have shown an increase in RR or PFS when compared to placebo, but in most cases this has not translated into an OS benefit. Predictive biomarkers are greatly needed to identify the subset of patients that may benefit from anti-angiogenic therapy or to identify patients likely to experience side effects, such as thrombosis and bleeding. A number of molecular signaling mediators of angiogenesis and inflammatory signaling have been investigated as potential biomarkers of anti-angiogenic therapy in lung cancer such as circulating VEGF ${ }^{64}$, intercellular adhesion molecule $(\mathrm{ICAM})^{64}, \mathrm{IL}^{2}{ }^{65}, \mathrm{IL}-8^{66}, \mathrm{IL}-12^{65}$, and IL-16 ${ }^{65}$, but no biomarker has yet been prospectively validated to correlate with outcomes.

\section{Conclusion}

As the field of lung cancer moves further into the personalized medicine age, it will be imperative that we target the entire milieu surrounding the tumor environment and not merely the mutations within the cancer cell itself. Preclinical models and selected clinical trials have shown benefits for targeting angiogenesis in lung cancer. Currently, bevacizumab is the only anti-angiogenic agent recommended by the NCCN for use in the treatment of advanced NSCLC. There is a significant knowledge deficit in the understanding of the molecular basis of anti-angiogenic therapy and the AEs seen with these agents. A more thorough understanding of both the mechanisms of benefit and AEs is needed to better predict who will benefit from this treatment strategy. Predictive biomarkers are needed to help select patients who will benefit most or be least likely to suffer from the toxicities associated with these drugs. 


\section{Acknowledgments}

Dr. Lammers is supported by the NIH (K12 CA 0906525). This work was supported by Boehringer Ingelheim Pharmaceuticals, Inc (BIPI). Editorial assistance was provided by Allison Michaelis, PhD, of MedErgy, which was contracted by BIPI for these services. The authors meet criteria for authorship as recommended by the International Committee of Medical Journal Editors (ICMJE), were fully responsible for all content and editorial decisions, and were involved at all stages of manuscript development. The authors received no compensation related to the development of the manuscript.

\section{Abbreviations:}

TKIs

VEGF

VEGFR

FGFR

PDGFR

c-KIT

FLT-3

RET

EGFR

PI3K

AKT

SRC

RAS

RAF

MEK

ERK tyrosine kinase inhibitors

vascular endothelial growth factor

vascular endothelial growth factor receptor

fibroblast growth factor receptor

platelet-derived growth factor receptor

stem cell factor receptor

fms-like tyrosine kinase 3

rearranged during transfection

epidermal growth factor receptor

phosphatidylinositol-3-kinase

protein kinase $\mathrm{B}$

v-src sarcoma viral oncogene homolog

retrovirus-associated DNA sequences

v-raf 1 murine leukemia viral oncogene homolog 1

mitogen activated protein kinase

extracellular signal-regulated kinase

\section{References}

1. American Cancer Society. Cancer Facts \& Figures, 2013. Atlanta, GA: American Cancer Society; 2013.

2. National Comprehensive Cancer Network. NCCN Clinical Practice Guidelines in Oncology ${ }^{\mathrm{TM}}$ NonSmall Cell Lung Cancer. V.3.2012 http://www.nccn.org/professionals/physician_gls/PDF/nscl.pdf.

3. Schiller JH, Harrington D, Belani CP, et al. Comparison of four chemotherapy regimens for advanced non-small-cell lung cancer. N Engl J Med 2002;346:92-98. [PubMed: 11784875]

4. Kris MG, Johnson BE, Kwiatkowski DJ, et al. Identification of driver mutations in tumor specimens from 1,000 patients with lung adenocarcinoma: The NCI's Lung Cancer Mutation Consortium (LCMC). J Clin Oncol 2011;29 (suppl). Abstract CRA7506.

5. Lucchi M, Mussi A, Fontanini G, et al. Small cell lung carcinoma (SCLC): the angiogenic phenomenon. Eur J Cardiothorac Surg 2002;21:1105-1110. [PubMed: 12048093] 
6. AVASTIN® (bevacizumab) Solution for intravenous infusion [package insert]. South San Francisco, CA: Genentech, Inc.; 2011.

7. Ferrara N, Hillan KJ, Gerber HP, et al. Discovery and development of bevacizumab, an anti-VEGF antibody for treating cancer. Nat Rev Drug Discov 2004;3:391-400. [PubMed: 15136787]

8. Johnson DH, Fehrenbacher L, Novotny WF, et al. Randomized phase II trial comparing bevacizumab plus carboplatin and paclitaxel with carboplatin and paclitaxel alone in previously untreated locally advanced or metastatic non-small-cell lung cancer. J Clin Oncol 2004;22:21842191. [PubMed: 15169807]

9. Sandler A, Gray R, Perry MC, et al. Paclitaxel-carboplatin alone or with bevacizumab for nonsmall-cell lung cancer. N Engl J Med 2006;355:2542-2550. [PubMed: 17167137]

10. Brahmer J, Dahlberg SE, Gray RJ, et al. Sex differences in outcome with bevacizumab therapy: analysis of patients with advanced-stage non-small cell lung cancer treated with or without bevacizumab in combination with paclitaxel and carboplatin in the Eastern Cooperative Oncology Group Trial 4599. J Thorac Oncol 2011;6:103-108. [PubMed: 21079521]

11. Ramalingam SS, Dahlberg SE, Langer CJ, et al. Outcomes for elderly, advanced-stage non smallcell lung cancer patients treated with bevacizumab in combination with carboplatin and paclitaxel: analysis of Eastern Cooperative Oncology Group Trial 4599. J Clin Oncol 2008;26:60-65. [PubMed: 18165641]

12. Reck M, von Pawel J, Zatloukal P, et al. Phase III trial of cisplatin plus gemcitabine with either placebo or bevacizumab as first-line therapy for nonsquamous non-small-cell lung cancer: AVAiL. J Clin Oncol 2009;27:1227-1234. [PubMed: 19188680]

13. Reck M, von Pawel J, Zatloukal P, et al. Overall survival with cisplatin-gemcitabine and bevacizumab or placebo as first-line therapy for nonsquamous non-small-cell lung cancer: results from a randomised phase III trial (AVAiL). Ann Oncol 2010;21:1804-1809. [PubMed: 20150572]

14. Patel JD, Hensing TA, Rademaker A, et al. Phase II study of pemetrexed and carboplatin plus bevacizumab with maintenance pemetrexed and bevacizumab as first-line therapy for nonsquamous non-small-cell lung cancer. J Clin Oncol 2009;27:3284-3289. [PubMed: 19433684]

15. Patel J, Socinski MA, Garon EB, et al. A randomized, open-label, phase 3, superiority study of pemetrexed $(\mathrm{Pem})+$ carboplatin $(\mathrm{Cb})+$ bevacizumab $(\mathrm{B})$ followed by maintenance Pem+B versus paclitaxel $(\mathrm{Pac})+\mathrm{Cb}+\mathrm{B}$ followed by maintenance B In patients (pts) with stage IIIB or IV nonsquamous non-small cell lung cancer (NS-NSCLC). J Thorac Oncol 2012;7(9 suppl 4):S336 Abstract LBPL1.

16. Hainsworth JD, Fang L, Huang JE, et al. BRIDGE: an open-label phase II trial evaluating the safety of bevacizumab + carboplatin/paclitaxel as first-line treatment for patients with advanced, previously untreated, squamous non-small cell lung cancer. J Thorac Oncol 2011;6:109-114. [PubMed: 21107290]

17. Besse B, Le Moulec S, Senellart J, et al. Final overall survival (OS) results of a noncomparative phase II study of bevacizumab plus first-line chemotherapy or second-line erlotinib in nonsquamous NSCLC patients with asymptomatic untreated brain metastases (BRAIN). J Clin Oncol 2013: 31; suppl; abstr 8059.

18. Spratlin JL, Cohen RB, Eadens M, et al. Phase I pharmacologic and biologic study of ramucirumab (IMC-1121B), a fully human immunoglobulin G1 monoclonal antibody targeting the vascular endothelial growth factor receptor-2. J Clin Oncol 2010;28:780-787. [PubMed: 20048182]

19. Camidge DR, Doebele RC, Ballas M, et al. Final results of a phase 2, open-label study of ramucirumab (IMC-1121B; RAM), an IGG1 MAb targeting VEGFR-2, with paclitaxel and carboplatin as first-line therapy in patients (pts) with stage IIIb/IV non-small cell lung cancer (NSCLC) (NCT00735696). Ann Oncol 2012;23(suppl 9):ix422-ix423. Abstract 1287P.

20. Doebele RC, Spigel D, Tehfe M, et al. A phase 2 randomized open-label study of ramucirumab (IMC 1121B; RAM) in combination with platinum-based chemotherapy in patients (pts) with recurrent or advanced non-small cell lung cancer (NSCLC): results from non-squamous (NSQ) PTS (NCT01160744). Ann Oncol 2012;23(suppl 9):ix408 Abstract 1245P.

21. DeRose P, Thorpe PE, Gerber DE. Development of bavituximab, a vascular targeting agent with immune-modulating properties, for lung cancer treatment. Immunotherapy 2011;3:933-944. [PubMed: 21843081] 
22. Dragnev KH, Attili SV, Gagua R, et al. A randomized, open-label, phase 2 trial of paclitaxel/ carboplatin with or without bavituximab in patients with previously untreated locally advanced or metastatic non-squamous non-small-cell lung cancer. J Thorac Oncol 2012;7(15):S241-S242. Abstract 180.

23. Lockhart AC, Rothenberg ML, Dupont J, et al. Phase I study of intravenous vascular endothelial growth factor trap, aflibercept, in patients with advanced solid tumors. J Clin Oncol 2010;28:207214. [PubMed: 19949018]

24. Leighl NB, Raez LE, Besse B, et al. A multicenter, phase 2 study of vascular endothelial growth factor trap (Aflibercept) in platinum- and erlotinib-resistant adenocarcinoma of the lung. $\mathbf{J}$ Thorac Oncol 2010;5:1054-1059. [PubMed: 20593550]

25. Ramlau R, Gorbunova V, Ciuleanu TE, et al. Aflibercept and docetaxel versus docetaxel alone after platinum failure in patients with advanced or metastatic non-small-cell lung cancer: a randomized, controlled phase III trial. J Clin Oncol 2012;30:3640-3647. [PubMed: 22965962]

26. Ferrara N, Davis-Smyth T. The biology of vascular endothelial growth factor. Endocr Rev 1997;18:4-25. [PubMed: 9034784]

27. Wilhelm SM, Carter C, Tang L, et al. BAY 43-9006 exhibits broad spectrum oral antitumor activity and targets the RAF/MEK/ERK pathway and receptor tyrosine kinases involved in tumor progression and angiogenesis. Cancer Res 2004;64:7099-7109. [PubMed: 15466206]

28. Blumenschein GR Jr., Gatzemeier U, Fossella F, et al. Phase II, multicenter, uncontrolled trial of single-agent sorafenib in patients with relapsed or refractory, advanced non-small-cell lung cancer. J Clin Oncol 2009;27:4274-4280. [PubMed: 19652055]

29. Spigel DR, Burris HA, Greco FA, et al. Randomized, double-blind, placebo-controlled, phase II trial of sorafenib and erlotinib or erlotinib alone in previously treated advanced, non-small-cell lung cancer. J Clin Oncol 2011;29:2582-2589. [PubMed: 21576636]

30. Scagliotti G, Novello S, von Pawel J, et al. Phase III study of carboplatin and paclitaxel alone or with sorafenib in advanced non-small-cell lung cancer. J Clin Oncol 2010;28:1835-1842. [PubMed: 20212250]

31. Paz-Ares LG, Biesma B, Heigener D, et al. Phase III, randomized, double-blind, placebocontrolled trial of gemcitabine/cisplatin alone or with sorafenib for the first-line treatment of advanced, nonsquamous non-small-cell lung cancer. J Clin Oncol 2012; 30:3084-3092. [PubMed: 22851564]

32. Abrams TJ, Lee LB, Murray LJ, et al. SU11248 inhibits KIT and platelet-derived growth factor receptor beta in preclinical models of human small cell lung cancer. Mol Cancer Ther 2003;2:471478. [PubMed: 12748309]

33. Socinski MA, Novello S, Brahmer JR, et al. Multicenter, phase II trial of sunitinib in previously treated, advanced non-small-cell lung cancer. J Clin Oncol 2008;26:650-656. [PubMed: 18235126]

34. Novello S, Scagliotti GV, Rosell R, et al. Phase II study of continuous daily sunitinib dosing in patients with previously treated advanced non-small cell lung cancer. Br J Cancer 2009;101:15431548. [PubMed: 19826424]

35. Scagliotti GV, Krzakowski M, Szczesna A, et al. Sunitinib Plus Erlotinib Versus Placebo Plus Erlotinib in Patients With Previously Treated Advanced Non-Small-Cell Lung Cancer: A Phase III Trial. J Clin Oncol 2012;30:2070-2078. [PubMed: 22564989]

36. Hilberg F, Roth GJ, Krssak M, et al. BIBF 1120: triple angiokinase inhibitor with sustained receptor blockade and good antitumor efficacy. Cancer Res 2008;68:4774-4782. [PubMed: 18559524]

37. Reck M, Kaiser R, Eschbach C, et al. A phase II double-blind study to investigate efficacy and safety of two doses of the triple angiokinase inhibitor BIBF 1120 in patients with relapsed advanced non-small-cell lung cancer. Ann Oncol 2011;22:1374-1381. [PubMed: 21212157]

38. Reck M, Kaiser R, Mellemgaard A, et al. Nintedanib (BIBF 1120) plus docetaxel in NSCLC patients progressing after first-line chemotherapy: LUME Lung 1, a randomized, double-blind phase III trial. J Clin Oncol 2013:31 (suppl; abstr LBA8011)

39. Hanna NH, Sullivan RN, Aren RO, et al. Lume-lung 2: A multicenter, randomized, double-blind, phase III study of nintedanib plus pemetrexed versus placebo plus pemetrexed in patients with 
advanced nonsquamous non-small cell lung cancer (NSCLC) after failure of first-line chemotherapy. J Clin Oncol 2013:31 (suppl; abstr 8034)

40. Wedge SR, Kendrew J, Hennequin LF, et al. AZD2171: a highly potent, orally bioavailable, vascular endothelial growth factor receptor-2 tyrosine kinase inhibitor for the treatment of cancer. Cancer Res 2005;65:4389-4400. [PubMed: 15899831]

41. Goss GD, Arnold A, Shepherd FA, et al. Randomized, double-blind trial of carboplatin and paclitaxel with either daily oral cediranib or placebo in advanced non-small-cell lung cancer: NCIC Clinical Trials Group BR24 study. J Clin Oncol 2010;28:49-55. [PubMed: 19917841]

42. Gadgeel SM, Ruckdeschel JC, Wozniak AJ, et al. Cediranib, a VEGF receptor 1, 2, and 3 inhibitor, and pemetrexed in patients (pts) with recurrent non-small cell lung cancer (NSCLC). J Clin Oncol 2011;29(suppl). Abstract 7564.

43. Polverino A, Coxon A, Starnes C, et al. AMG 706, an oral, multikinase inhibitor that selectively targets vascular endothelial growth factor, platelet-derived growth factor, and kit receptors, potently inhibits angiogenesis and induces regression in tumor xenografts. Cancer Res 2006;66:8715-8721. [PubMed: 16951187]

44. Blumenschein GR Jr., Kabbinavar F, Menon H, et al. A phase II, multicenter, open-label randomized study of motesanib or bevacizumab in combination with paclitaxel and carboplatin for advanced nonsquamous non-small-cell lung cancer. Ann Oncol 2011;22:2057-2067. [PubMed: 21321086]

45. Scagliotti GV, Vynnychenko I, Park K, et al. International, randomized, placebo-controlled, double-blind phase III study of motesanib plus carboplatin/paclitaxel in patients with advanced nonsquamous non-small-cell lung cancer: MONET1. J Clin Oncol 2012;30:2829-2836. [PubMed: 22753922]

46. Kumar R, Knick VB, Rudolph SK, et al. Pharmacokinetic-pharmacodynamic correlation from mouse to human with pazopanib, a multikinase angiogenesis inhibitor with potent antitumor and antiangiogenic activity. Mol Cancer Ther 2007;6:2012-2021. [PubMed: 17620431]

47. Spigel D, Burris HA, Greco FA, et al. A randomized phase II study of pazopanib or placebo in combination with erlotinib in patients with advanced non-small-cell lung cancer. J Thorac Oncol 2012;7(15):S208 Abstract 13.

48. Rugo HS, Herbst RS, Liu G, et al. Phase I trial of the oral antiangiogenesis agent AG-013736 in patients with advanced solid tumors: pharmacokinetic and clinical results. J Clin Oncol 2005;23:5474-5483. [PubMed: 16027439]

49. Schiller JH, Larson T, Ou SH, et al. Efficacy and safety of axitinib in patients with advanced nonsmall-cell lung cancer: results from a phase II study. J Clin Oncol 2009;27:3836-3841. [PubMed: 19597027]

50. Belani CP, Yamamoto N, Bondarenko I, et al. A randomized phase II study of axitinib in combination with pemetrexed/cisplatin (pem/cis) as first-line therapy for nonsquamous non-small cell lung cancer (NSCLC). J Clin Oncol 2012;30(suppl). Abstract 7551.

51. Twelves C, Chmielowska E, Havel L, et al. Randomised phase II study of axitinib or bevacizumab combined with paclitaxel/carboplatin (PAC/CARB) as first-line therapy for patients (pts) with advanced non-small cell lung cancer (NSCLC). Ann Oncol 2012;23(suppl 9):ix409 Abstract 1246P.

52. Morabito A, Piccirillo MC, Falasconi F, et al. Vandetanib (ZD6474), a dual inhibitor of vascular endothelial growth factor receptor (VEGFR) and epidermal growth factor receptor (EGFR) tyrosine kinases: current status and future directions. Oncologist 2009;14:378-390. [PubMed: 19349511]

53. De Boer RH, Arrieta O, Yang CH, et al. Vandetanib plus pemetrexed for the second-line treatment of advanced non-small-cell lung cancer: a randomized, double-blind phase III trial. J Clin Oncol 2011;29:1067-1074. [PubMed: 21282537]

54. Herbst RS, Sun Y, Eberhardt WE, et al. Vandetanib plus docetaxel versus docetaxel as second-line treatment for patients with advanced non-small-cell lung cancer (ZODIAC): a double-blind, randomised, phase 3 trial. Lancet Oncol 2010;11:619-626. [PubMed: 20570559]

55. Lee JS, Hirsh V, Park K, et al. Vandetanib versus placebo in patients with advanced non-small-cell lung cancer after prior therapy with an epidermal growth factor receptor tyrosine kinase inhibitor: 
a randomized, double-blind phase III trial (ZEPHYR). J Clin Oncol 2012;30:1114-1121. [PubMed: 22370318]

56. Natale RB, Thongprasert S, Greco FA, et al. Phase III trial of vandetanib compared with erlotinib in patients with previously treated advanced non-small-cell lung cancer. J Clin Oncol 2011;29:1059-1066. [PubMed: 21282542]

57. Albert DH, Tapang P, Magoc TJ, et al. Preclinical activity of ABT-869, a multitargeted receptor tyrosine kinase inhibitor. Mol Cancer Ther 2006;5:995-1006. [PubMed: 16648571]

58. Tan EH, Goss GD, Salgia R, et al. Phase 2 trial of linifanib (ABT-869) in patients with advanced non-small cell lung cancer. J Thorac Oncol 2011;6:1418-1425. [PubMed: 21597387]

59. Hori K, Saito S, Kubota K. A novel combretastatin A-4 derivative, AC7700, strongly stanches tumour blood flow and inhibits growth of tumours developing in various tissues and organs. $\mathrm{Br} \mathrm{J}$ Cancer 2002;86:1604-1614. [PubMed: 12085211]

60. von Pawel J, Gorbounova V, Reck M, et al. DISRUPT: a randomized phase 2 trial of ombrabulin (AVE8062) combined with a taxane-platinum regimen in the first-line treatment of metastatic nonsmall cell lung cancer (NSCLC). Ann Oncol 2012;23(suppl 9):ix410 Abstract 1250P.

61. McKeage MJ, Baguley BC. Disrupting established tumor blood vessels: An emerging therapeutic strategy for cancer. Cancer 2010;116:1859-1871. [PubMed: 20166210]

62. McKeage MJ, von Pawel J, Reck M. Randomised phase II study of ASA404 combined with carboplatin and paclitaxel in previously untreated advanced non-small cell lung cancer. Br J Cancer 2008;99:2006-2012. [PubMed: 19078952]

63. Lara PN, Douillard JY, Nakagawa K, et al. Randomized phase III placebo-controlled trial of carboplatin and paclitaxel with or without the vascular disrupting agent vadimezan (ASA404) in advanced non-small cell lung cancer. J Clin Oncol 2011;29:2965-2971. [PubMed: 21709202]

64. Dowlati A, Gray R, Sandler AB, et al. Cell adhesion molecules, vascular endothelial growth factor, and basic fibroblast growth factor in patients in non-small cell lung cancer treated with chemotherapy with or without bevacizumab-an Eastern Cooperative Oncology Group Study. Clin Cancer Res. 2008;14:1407-1412. [PubMed: 18316562]

65. Nikolinakos PG, Altorki N, Yankelevitz D, et al. Plasma cytokine and angiogenic factor profiling identifies markers associated with tumor shrinkage in early-stage non-small cell lung cancer patients treated with pazopanib. Cancer Res. 2010;70:2171-2179. [PubMed: 20215520]

66. Hanrahan EO, Lin HY, Kim ES, et al. Distinct patterns of cytokine and angiogenic factor modulation and markers of benefit for vandetanib and/or chemotherapy in patients with non-smallcell lung cancer. J Clin Oncol. 2010;28:193-201. [PubMed: 19949019] 


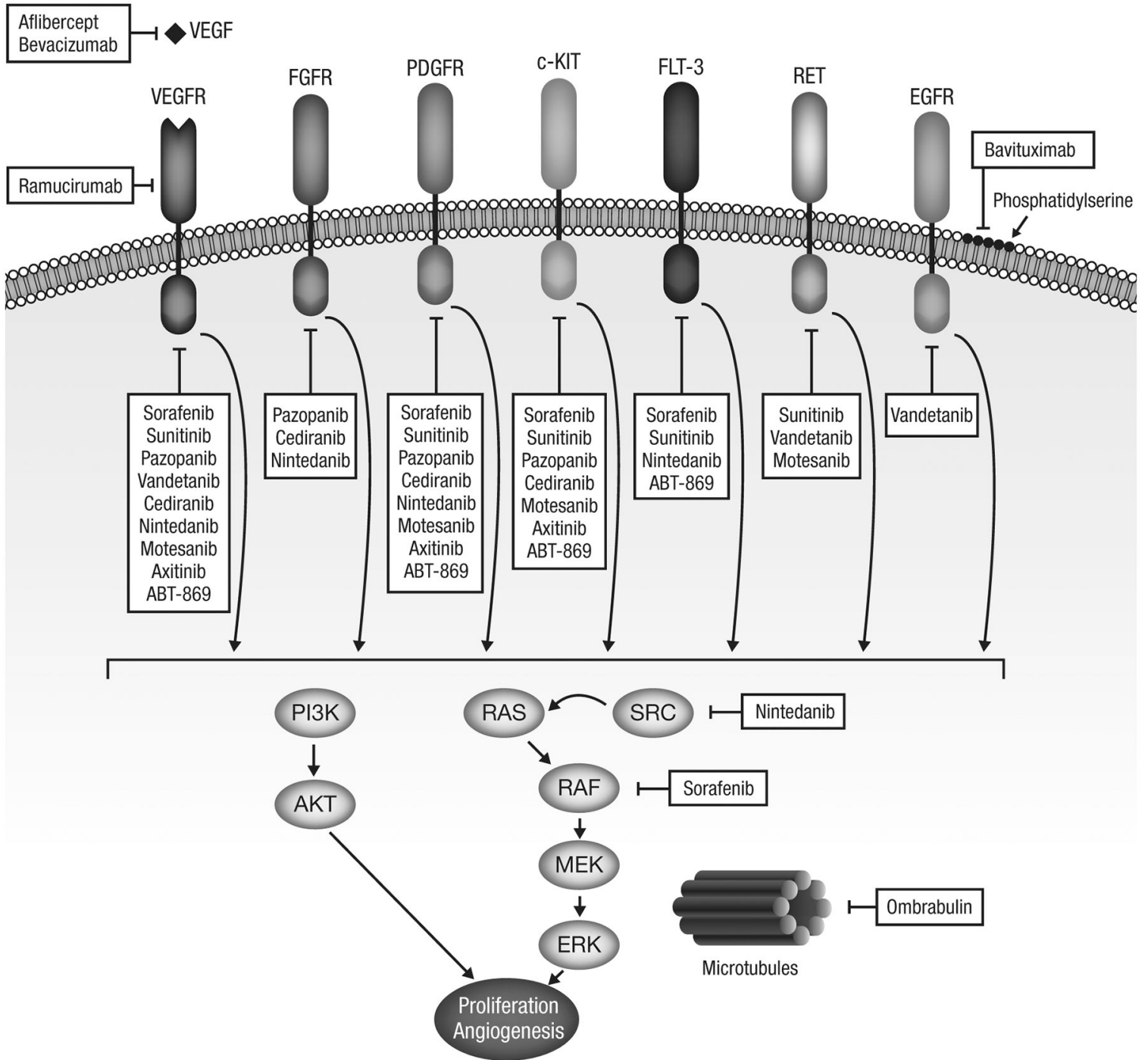

Figure 1. Targeting angiogenesis in lung cancer.

Receptors and downstream signaling pathways involved in angiogenesis and sites of action of anti-angiogenic antibody-based therapies and multi-targeted TKIs. 


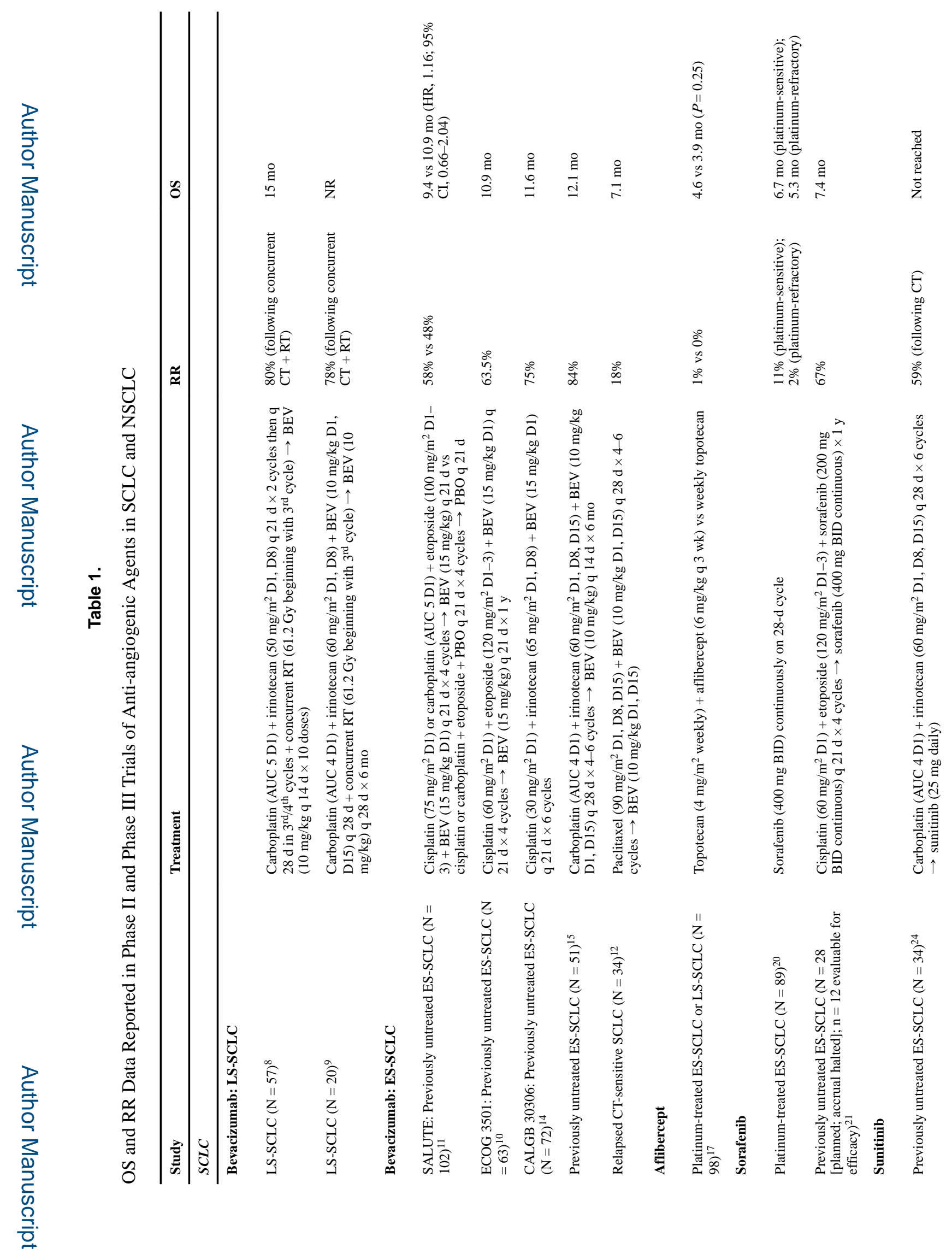

J Natl Compr Canc Netw. Author manuscript; available in PMC 2019 July 15. 


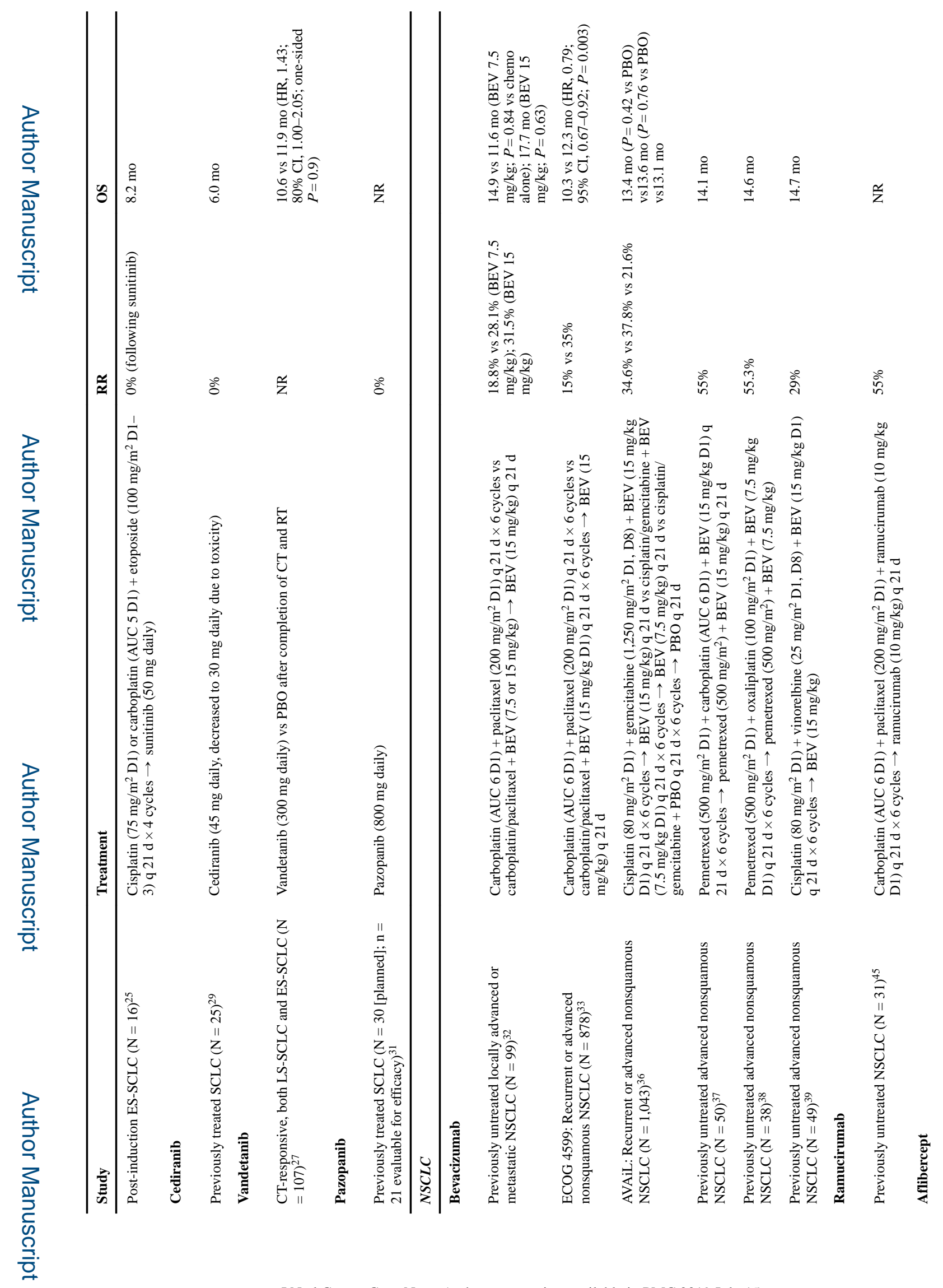

J Natl Compr Canc Netw. Author manuscript; available in PMC 2019 July 15. 


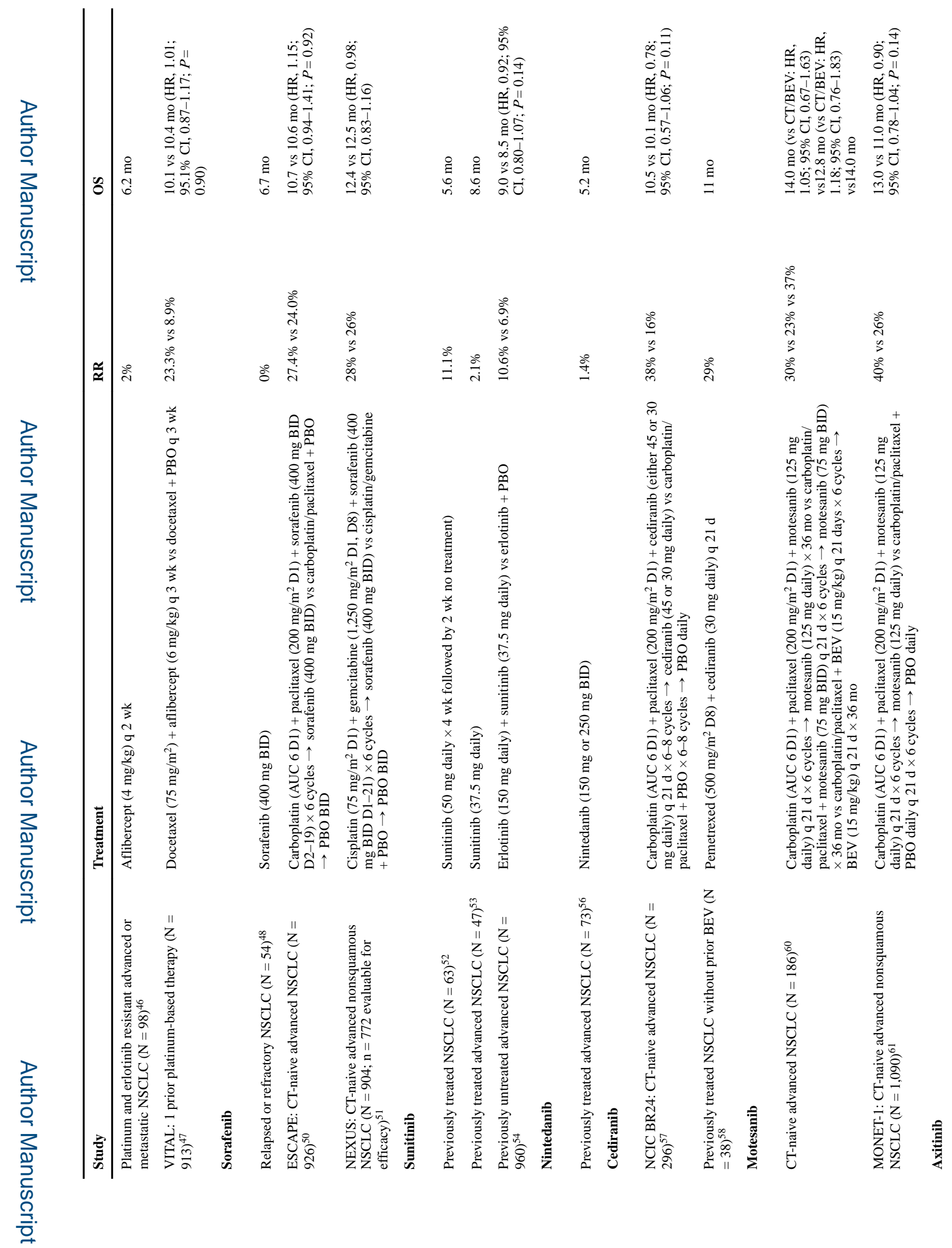

J Natl Compr Canc Netw. Author manuscript; available in PMC 2019 July 15. 


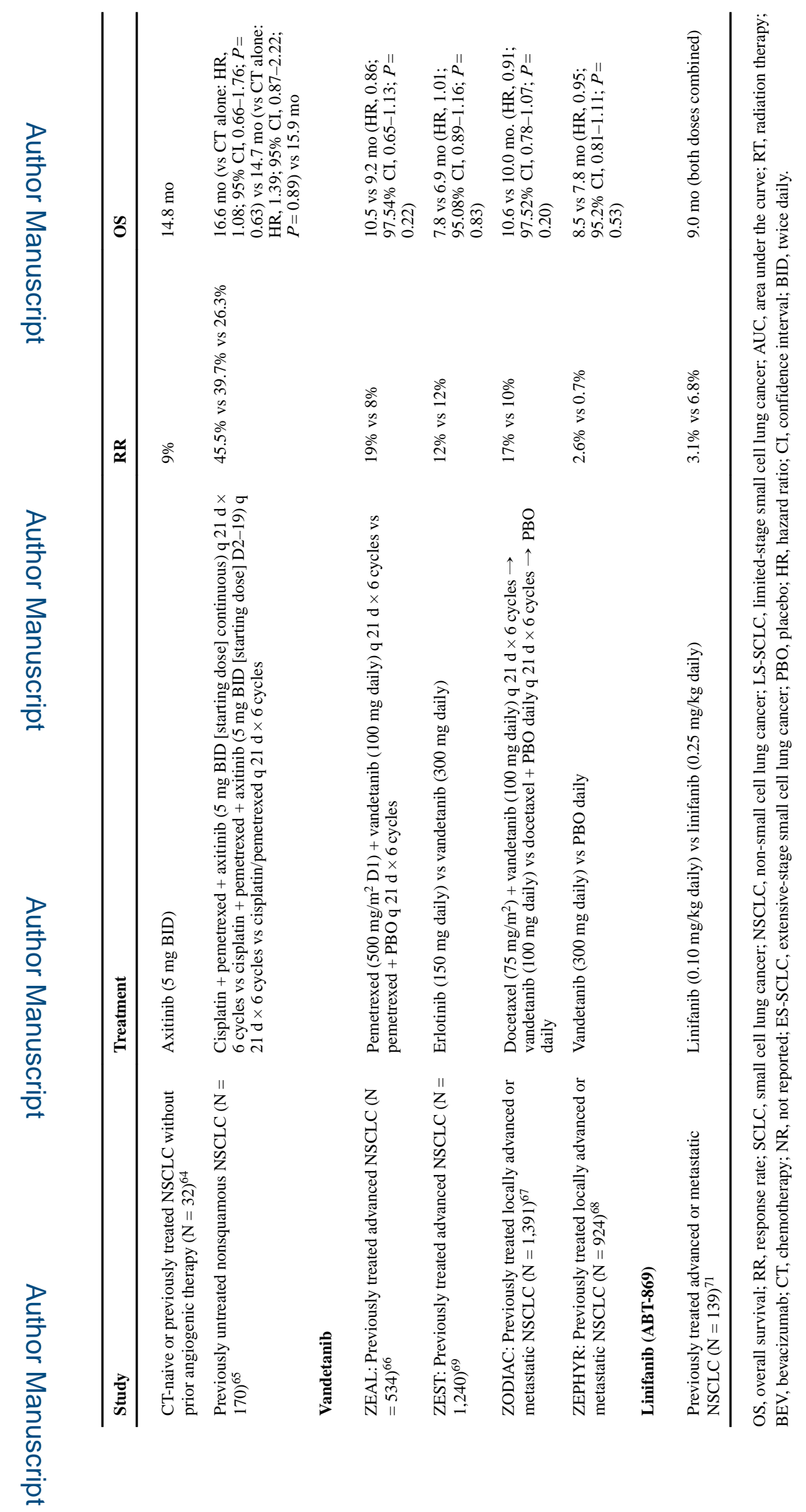

J Natl Compr Canc Netw. Author manuscript; available in PMC 2019 July 15. 


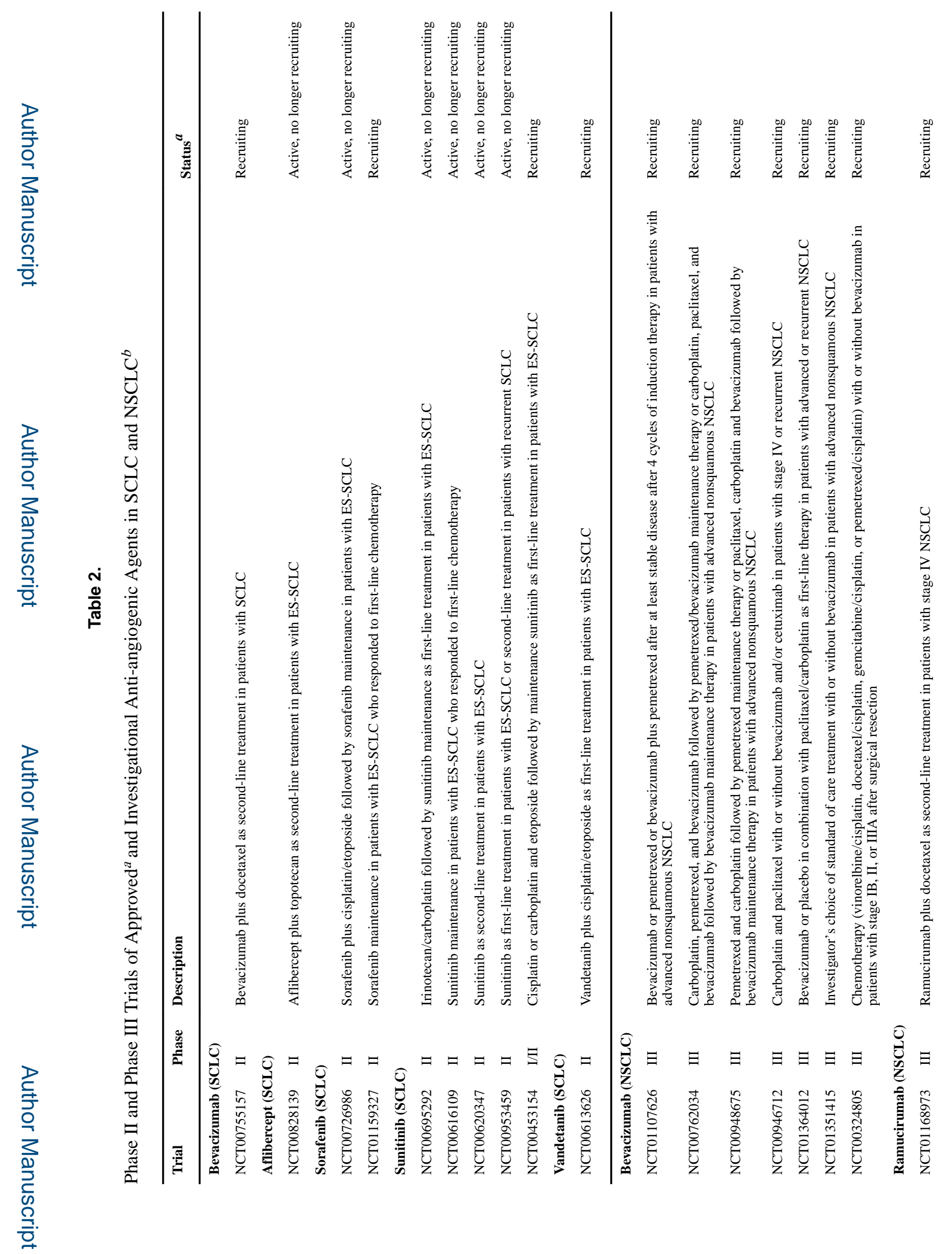

J Natl Compr Canc Netw. Author manuscript; available in PMC 2019 July 15. 


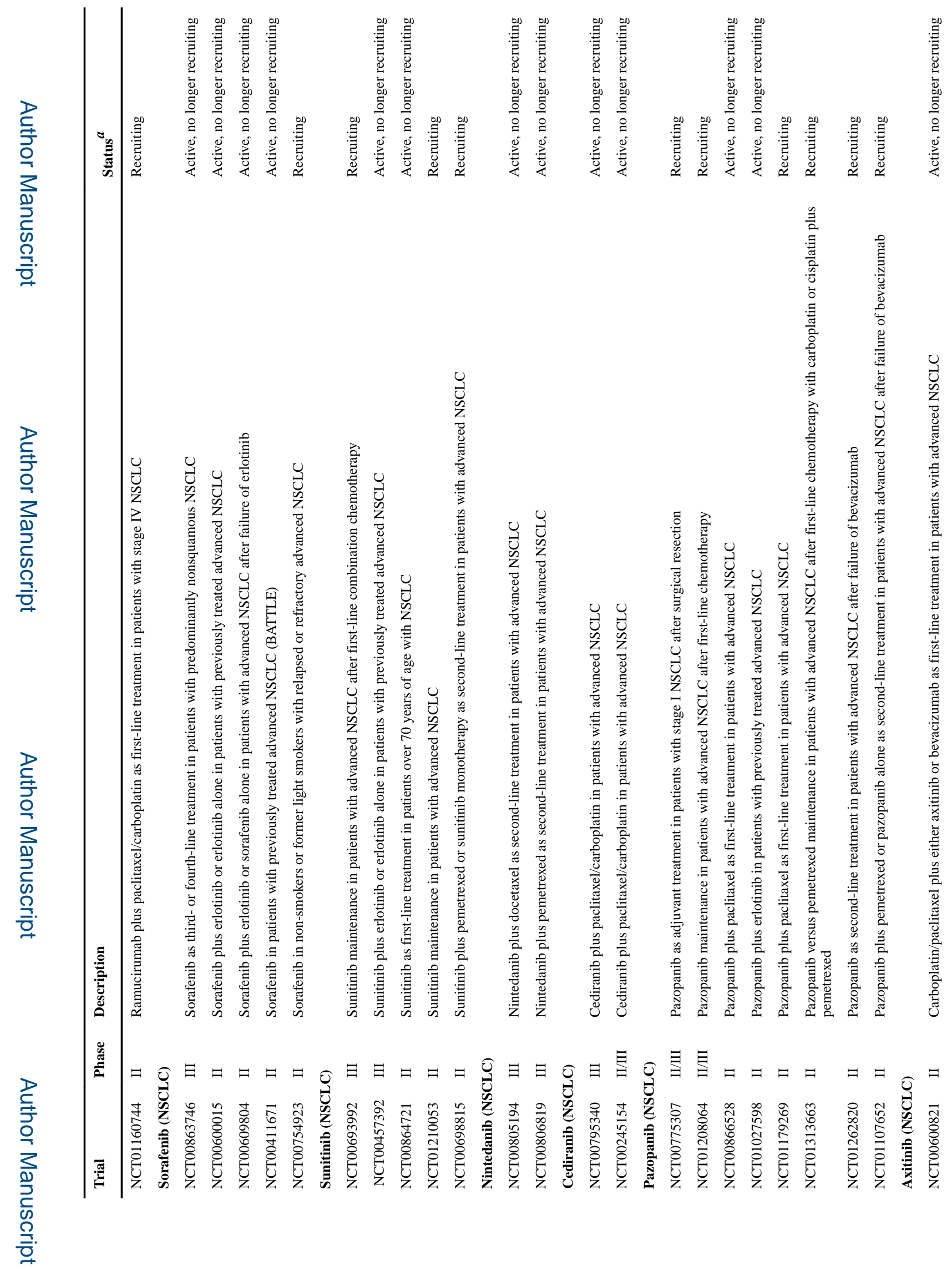

J Natl Compr Canc Netw. Author manuscript; available in PMC 2019 July 15. 


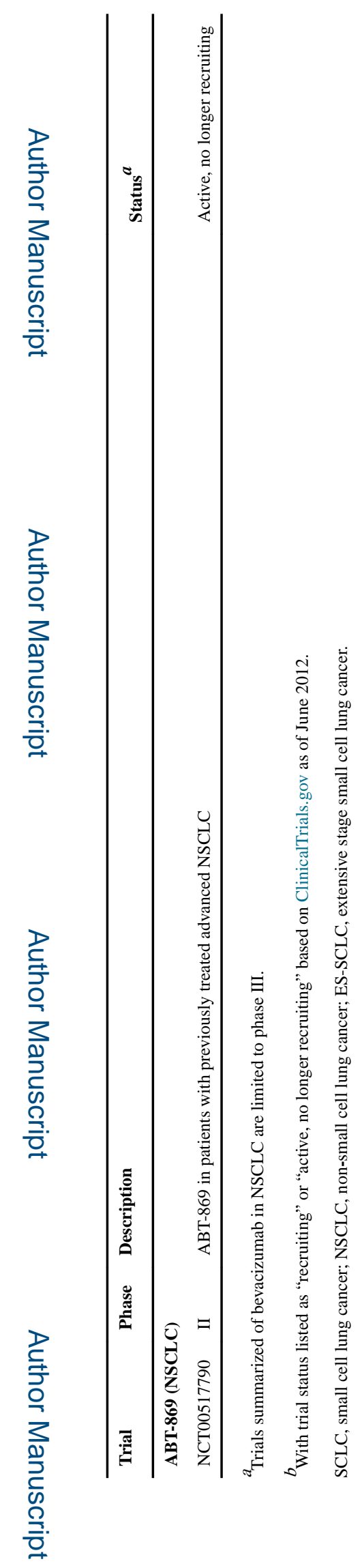

J Natl Compr Canc Netw. Author manuscript; available in PMC 2019 July 15. 